\title{
Monitoring of fibrinolytic system activity with plasminogen, D-dimers and FDP in primary total knee arthroplasty (TKA) after topical, intravenous or combined administration of tranexamic acid
}

\author{
Jiri Lostaka , Jiri Gallo ${ }^{\mathrm{a}}$, Ludek Slavik ${ }^{\mathrm{b}}$, Jana Zapletalovac', Lubos Balaz ${ }^{\mathrm{a}}$
}

\begin{abstract}
Aim. We assessed various ways of tranexamic acid (TXA) administration on the fibrinolytic system. Blood loss, transfusions, drainage and haematoma were secondary outcomes.

Methods. In this prospective study, we examined 100 patients undergoing primary total knee arthroplasty (TKA) between June and November 2018. Patients were randomly assigned to 4 groups according to the following TXA regimens: 1) loading dose $15 \mathrm{mg}$ TXA/kg single intravenous administration applied at initiation of anesthesia (IV1);2) loading dose $15 \mathrm{mg}$ TXA/kg + additional dose $15 \mathrm{mg} \mathrm{TXA} / \mathrm{kg} 6 \mathrm{~h}$ after the first application of TXA (IV2); 3) IV1 regime in combination with a local wash of $2 \mathrm{~g}$ of TXA in $50 \mathrm{~mL}$ of saline (COMB); 4) topical administration of $2 \mathrm{~g}$ of TXA in 50 $\mathrm{mL}$ of saline (TOP).

Results. Systemic fibrinolysis interference was insignificant in all of the regimens; we did not detect significant differences between IV1, IV2 and COMB in the monitored parameters within the elapsed time after the TKA; IV regimes had the lowest total drainage blood loss; the lowest blood loss was associated with the IV1 and IV2 regimens (IV1, IV2 $<\mathrm{COMB}<\mathrm{TOP}$ ); the lowest incidence of haematomas was in patients treated with TXA topically (i.e., in COMB + TOP). Conclusion. The largest antifibrinolytic effect was associated with intravenous administration of TXA. In terms of blood loss, intravenously administered TXA can interfere with the processes associated with the formation of the fibrin plug more efficiently than the simple washing of wound surfaces with TXA.
\end{abstract}

Key words: tranexamic acid, total knee arthroplasty, topical application, intravenous administration, combined administration, plasminogen, D-dimers, FDP, blood loss

Received: April 25, 2019; Revised: July 5, 2019; Accepted: July 17, 2019; Available online: September 16, 2019 https://doi.org/10.5507/bp.2019.034

(c) 2020 The Authors; https://creativecommons.org/licenses/by/4.0/

${ }^{a}$ Department of Orthopaedics, University Hospital Olomouc and Faculty of Medicine and Dentistry, Palacky University Olomouc, Czech Republic

${ }^{b}$ Department of Haemato-Oncology, University Hospital Olomouc and Faculty of Medicine and Dentistry, Palacky University Olomouc, Czech Republic

'Department of Medical Biophysics, Faculty of Medicine and Dentistry, Palacky University Olomouc, Czech Republic Corresponding author: Jiri Gallo, e-mail:jiri.gallo@volny.cz

\section{INTRODUCTION}

Tranexamic acid (TXA) is a synthetic amino acid derivative of lysine that inhibits the conversion of plasminogen to plasmin by blocking the binding site of plasminogen to a fibrin molecule. At higher concentrations, TXA can also directly inhibit plasmin activity ${ }^{1,2}$. It is also believed that TXA has an anti-inflammatory effect (inhibition of plasmin-mediated complement, monocyte and neutrophil inhibition $)^{3}$. Compared with other antifibrinolytics, the effect of TXA is more potentiated by factor $\mathrm{X}\left(\mathrm{ref}^{4}{ }^{4}\right)$.

TXA has gradually become a routine part of perioperative care in primary total arthroplasty of the hips and knees $^{5,6}$. Therefore, the clinical utility of this intervention is unquestionable and is supported by several meta-analyses of RCTs (randomized clinical trials) for primary hips and knees ${ }^{7-11}$. TXA can be administered by the intravenous route, orally or topically into the joint ${ }^{1}$. Most studies were performed with systemic administration of TXA ( ref. $\left.^{1}\right)$.
More recently, TXA has been combined with intra-articular (topical) and intravenous administration ${ }^{12,13}$. Topical administration is justified by an effort to transfer the maximum effect to the target area and to avoid systemic effects on the fibrinolytic system ${ }^{14}$. Excessive interference with the mechanism of fibrinolysis may lead to an increased risk of intravascular closures, for example, in the form of thromboembolic disease (VTE), a dreaded complication of joint replacement surgery.

The aim of our study was to evaluate the effect of 4 regimens of TXA administration on systemic fibrinolysis. Diagnosis of hyperfibrinolysis (HF) is not easy as there are currently no specific tests that allow for its assessment available. In our study, we measured HF according to plasma levels in combination with determination of HF products, such as D-dimers (DDIMs) and fibrinfibrinogen degradation products (FDP). These tests are sensitive enough for monitoring within the time available in all coagulation laboratories. 


\section{MATERIAL AND METHODS}

\section{Patients}

In this prospective study, we examined a total of 100 consecutive patients indicated for primary TKA at our clinic. They were assigned to any of the four study protocol groups based on the method of TXA administration. Each group comprised 25 patients. The baseline criteria for inclusion in the study were normal pre-operative blood count (haemoglobin, thrombocytes) and blood coagulation (INR, Quick, aPTT) parameters. Patients with any history of a blood clotting disorder, of VTE, or who had a more severe kidney disease and/or suffered from seizures were excluded. The patient groups displayed the same basic characteristics (Table 1). Prior to enrollment, patients signed a specific informed consent form. The Ethical Committee for Faculty of Medicine and Dentistry, Palacky University Olomouc and University Hospital Olomouc approved this study in accordance with the Helsinki Declaration (registration number 38/19).

\section{Data Collection}

The data collection was performed prospectively according to a previuosly agreed protocol. Medical data was collected by the physician during patient hospitalisation.

\section{Perioperative regime}

Patient preparation for TKA surgery started on the day of admission, i.e., the day before surgery. In terms of VTE prevention, we applied either low molecular weight heparin (Fraxiparine, Glaxosmithkline), which we administered subcutaneously for the first time $12 \mathrm{~h}$ before surgery at the dose recommended by the manufacturer, or the oral administration of Rivaroxaban (Xarelto, Bayer) 6 to $8 \mathrm{~h}$ after surgery. To prevent post-operative infection, we primarily used the Azepo third-generation cephalosporin antibiotic (Sandoz) at a dose of $1 \mathrm{~g}$ i.v., substituting it with $600 \mathrm{mg}$ i.v. of Clindamycin (Pfizer) in case of allergy. The antibiotic was administered intravenously 30-60 min before surgery. We applied the next two doses after $8 \mathrm{~h}$.

\section{Operation procedure and implant}

The surgery was performed under general or spinal anesthesia. For all patients, we operated from the middle skin incision and medial parapatelar approach. Bleeding was continuously stopped during TKA with electrocoagulation. A tourniquet was only applied upon cementing (usually 10-15 min). All operations were performed by experienced surgeons. We solely used cemented posterior cruciate retaining implants, respectively posteriorstabilised implants (PS version).

\section{Study design}

Each group had a precisely defined TXA protocol and dosing schedule. The first group (IV1) included patients who were administered TXA via a single intravenous dose (15 mg TXA $/ \mathrm{kg}$ ) at initiation of anaesthesia. In the second group of patients (IV2), TXA was administered in two intravenous doses ( $15 \mathrm{mg}$ TXA/ $\mathrm{kg}$ ), at the initiation of anaesthesia and 6 hours after the start of surgery. The third group of patients (TOP) was administered TXA topically by rinsing with a diluted solution containing $2 \mathrm{~g}$ of TXA in $50 \mathrm{~mL}$ of saline. In the fourth group (COMB), TXA was administered in combination, the first $15 \mathrm{mg}$ TXA/ kg intravenously at the initiation of anaesthesia, and the second dose was topically rinsed with $2 \mathrm{~g}$ of TXA in $50 \mathrm{~mL}$ of saline at the end of the operation. For all patients, we left the drain closed for 1 hour after surgery. The TXA protocols are listed in Table 2.

The primary objective of the study was to determine how the different methods of TXA administration influence the systemic parameters of fibrinolysis. A secondary objective was to compare which of the tested regimens resulted in the lowest postoperative blood loss, respectively to the lowest consumption of blood transfusions and complication rates.

\section{Monitoring parameters}

For each patient, we took the plasminogen, D-dimers, FDP and blood count according to the protocol. The first sample was performed by an anaesthesiologist at the start

Table 1. Comparison of TXA groups in patient demographic and clinical characteristics.

\begin{tabular}{|c|c|c|c|c|c|}
\hline & $\begin{array}{c}\text { IV1 } \\
(n=25)\end{array}$ & $\begin{array}{c}\mathrm{IV} 2 \\
(\mathrm{n}=25)\end{array}$ & $\begin{array}{l}\text { COMB } \\
(\mathrm{n}=25)\end{array}$ & $\begin{array}{c}\text { TOP } \\
(n=25)\end{array}$ & $P$ \\
\hline $\begin{array}{l}\text { Primary/secondary } \\
\text { osteoarthritis }\end{array}$ & $23 / 2(92 \% / 8 \%)$ & $23 / 2(92 \% / 8 \%)$ & $21 / 4(84 \% / 16 \%)$ & $21 / 4(84 \% / 16 \%)$ & 0.744 \\
\hline Sex (male/female) & $8 / 17(32 \% / 68 \%)$ & $10 / 15(40 \% / 60 \%)$ & $13 / 12(52 \% / 48 \%)$ & $13 / 12(52 \% / 48 \%)$ & 0.402 \\
\hline Average age (years) & $71.2 \pm 7.3$ & $68.6 \pm 6.4$ & $67.9 \pm 7.2$ & $70.1 \pm 7.6$ & 0.348 \\
\hline $\begin{array}{l}\text { Body mass index }\left(\mathrm{kg} / \mathrm{m}^{2}\right) \text {, } \\
\text { (median, min-max) }\end{array}$ & $31.2(25.2-41.9)$ & $30.7(21.3-45.1)$ & $32.0(21.3-45.1)$ & $31.2(24.8-44.1)$ & 0.884 \\
\hline ASA score (I/II/II-III/III) & $0 / 23 / 0 / 2$ & $0 / 21 / 0 / 4$ & $2 / 17 / 3 / 3$ & $0 / 19 / 0 / 6$ & 0.087 \\
\hline $\begin{array}{l}\text { Anticoagulation prophylaxis } \\
\text { (LMWH vs. DOAC) }\end{array}$ & $17 / 8(68 \% / 32 \%)$ & $16 / 9(64 \% / 36 \%)$ & $14 / 11(56 \% / 44 \%)$ & $20 / 5(80 \% / 20 \%)$ & 0.369 \\
\hline IKDC classification & $10 / 12 / 3$ & $9 / 14 / 2$ & $11 / 12 / 2$ & $10 / 15 / 0$ & 0.617 \\
\hline
\end{tabular}

n - number of patients; $P$ - significance value, $<0.05$ was considered statistically significant; LMWH - low molecular weight heparin; DOAC - direct oral anticoagulants; IKDC - International Knee Documentation Committee; ASA - American Society of Anesthesiologists; IV1 - intravenous administration - single dose; IV2 - intravenous administration - two doses; COMB - combination of intravenous and topical administration; TOP - only topical administration; TXA - tranexamic acid. 
Table 2. TXA administration protocols.

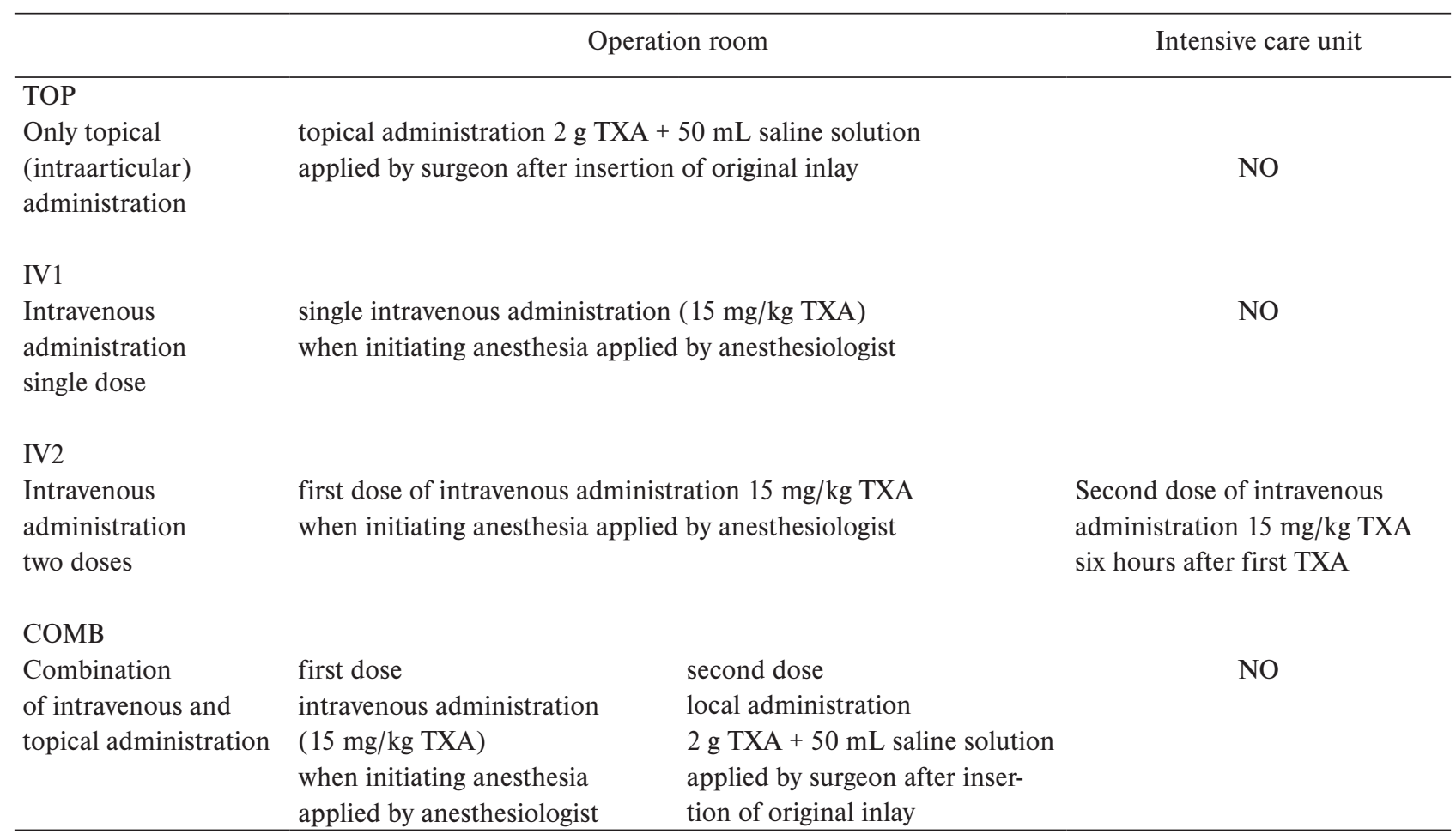

TXA - tranexamic acid

of anaesthesia, just before applying TXA. Other samples were taken 3,6 and $12 \mathrm{~h}$ after the start of surgery.

\section{Blood loss including hidden blood loss}

We recorded the amount of blood loss during TKA and postoperative drainage and, respectively, the number of blood transfusions. Hidden blood loss was calculated according to a formula that includes peroperative and postoperative loss based on gender and patient weight ${ }^{15}$.

\section{Haemoglobin, haematocrit}

The blood count (haemoglobin, haematocrit, erythrocytes, platelets) was routinely examined on the Sysmex XN 3000 analyser (Sysmex, Kobe, Japan) at the intervals listed above, additionally on the $1^{\text {st }}$ and $2^{\text {nd }}$ postoperative days and, in some patients, also on the $3^{\text {rd }}$ postoperative day. By having pre-operative baseline values, it was possible to determine the decrease in haemoglobin (the difference between pre-operative and postoperative value) induced by surgery.

\section{Fibrinolysis monitoring}

Plasminogen (Plg) was detected by the chromogenic method. Plg is measured by its specific activation upon the addition of excess streptokinase and fibrinogen without plasminogen. The plasminogen-streptokinase complex has a plasmin-like activity that specifically cleaves a plasmid-specific substrate SPm41 releasing a characteristic para-nitroaniline stain (pNA) detected at $405 \mathrm{~nm}$. The rate of increase in absorbance is directly proportional to plasma plasminogen ${ }^{16-18}$.

D-dimer is formed by the cleavage of cross-linked fi- brin fibres by the action of plasmin. D-dimer is detected by a suspension of polystyrene latex particles of uniform size coated with the $F\left(a b^{\prime}\right) 2$ monoclonal antibody fragment highly specific for the D-Dimer domain. The degree of agglutination is directly proportional to the concentration of D-dimer in the sample and is determined by measuring the decrease in transmitted light caused by aggregates (turbidimetric immunoassay) ${ }^{19-21}$.

FDP is detected with a suspension of polystyrene latex particles of uniform size coated with a monoclonal antibody highly specific for the $\mathrm{D}$ fragment included in fibrin/ fibrinogen soluble derivatives. The degree of agglutination is directly proportional to the concentration of FDP in the sample and is determined by measuring the decrease of transmitted light at the wavelength of $671 \mathrm{~nm}$ due to aggregates (turbidimetric immunoassay) ${ }^{22,23}$.

\section{Haematoma incidence, wound secretion after the $4^{\text {th }}$ postoperative day}

We monitored the surgical wound healing and the presence and localisation of the haematoma. Major wound disturbances that persisted after the $4^{\text {th }}$ postoperative day were recorded.

\section{Statistical analysis}

The distribution of quantitative data was verified by the Shapiro-Wilk's normality test. Data that had a normal distribution was presented using mean and standard deviation, and variance analysis (ANOVA) was used to verify differences between the groups. If the data did not have a normal distribution, it was described using median, minimum and maximum values. Comparison of the groups 
Table 3. Level of D-dimers and fibrin degradation products 3, 6 and $12 \mathrm{~h}$ after the start of surgery.

\begin{tabular}{lccccc}
\hline D-dimers & IV1 & IV2 & COMB & TOP & $P$ \\
\hline preoperative & $537(157-15,239)$ & $402(139-888)$ & $616(136-2,653)$ & $575(167-3,433)$ & 0.020 \\
3 h after the start of surgery & $1,638(376-6,780)$ & $1,409(411-5,516)$ & $1,275(738-15,886)$ & $4,773(1,139-22,443)$ & $<0.0001$ \\
6 h after the start of surgery & $3,897(592-16,249)$ & $3,610(799-13,814)$ & $1,617(768-15,792)$ & $7,014(1,218-32,691)$ & 0.001 \\
12 h after the start of surgery & $4,970(836-26,270)$ & $1,925(599-13,159)$ & $2,026(860-21,213)$ & $4,480(1,031-25,338)$ & 0.013 \\
\hline FDP & IV1 & IV2 & COMB & TOP & $P$ \\
\hline preoperative & $4.6(2.1-33.6)$ & $4.1(2.5-5.2)$ & $4.6(2.7-10.3)$ & $4.7(2.2-11.3)$ & 0.118 \\
3 h after the start of surgery & $7.0(3.0-18.0)$ & $6.6(3.5-13.2)$ & $6.6(3.1-38.9)$ & $14.0(4.9-58.7)$ & $<0.0001$ \\
6 h after the start of surgery & $11.0(3.5-39.2)$ & $10.5(4.6-31.9)$ & $7.6(5.3-36.8)$ & $19.8(5.2-98.4)$ & 0.002 \\
12 h after the start of surgery & $13.7(4.6-67.9)$ & $9.1(4.1-26.0)$ & $8.9(5.3-57.5)$ & $12.3(5.5-66.1)$ & 0.016 \\
\hline
\end{tabular}

$P$ - significance value Kruskal-Wallis test, median (min-max); IV1 - intravenous administration - single dose; IV2 - intravenous administration - two doses; COMB - combination of intravenous and topical administration; TOP - only topical administration; TKA - total knee arthroplasty; FDP - fibrin degradation products
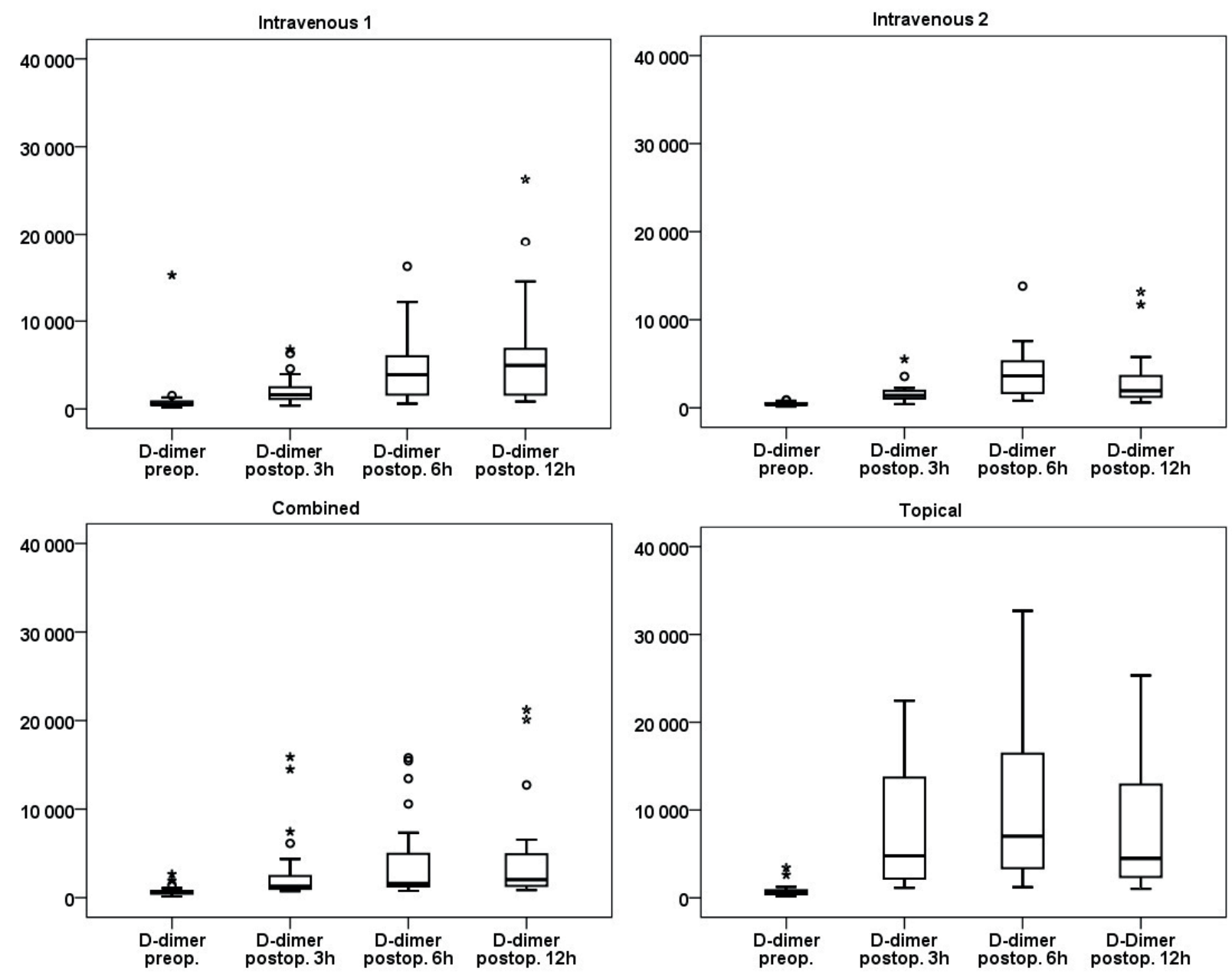

Fig. 1. Comparison of the level of D-dimers for each TXA group after 3, 6 and $12 \mathrm{~h}$ after the start of the surgery. TXA - tranexamic acid; TKA - total knee arthroplasty

was performed by the non-parametric Kruskal-Wallis test and post-hoc Dunn test. Qualitative data was described using absolute and relative frequencies and analysed with Fisher's exact test. The dependence between the quantitative parameters was assessed using Spearman's correlation analysis. The Mann-Whitney $U$ test was used to analyse the dependence between measured quantitative parameters and sex, type of arthrosis or selected VTE prevention. The IBM SPSS Statistics version 22 software was used for statistical analysis. All tests were performed at a statistical significance level of 0.05 . 
Table 4. Comparison of perioperative blood loss between patient TXA groups.

\begin{tabular}{|c|c|c|c|c|c|}
\hline & IV1 & IV2 & COMB & TOP & $P$ \\
\hline Total blood loss to drains (mL) & $300(0-1,370)$ & $350(0-1,000)$ & $650(0-1,500)$ & $670(0-1,070)$ & 0.002 \\
\hline Hidden blood loss (mL) & $228(28-619)$ & $203(13-754)$ & $328(9-690)$ & $349(84-596)$ & 0.007 \\
\hline Blood loss during TKA & $300(200-500)$ & $300(200-800)$ & $300(200-700)$ & $300(100-650)$ & 0.959 \\
\hline $\begin{array}{l}\text { Total blood loss }(\mathrm{mL}), \\
\text { (peroper. + drainage) }\end{array}$ & $600(200-1,720)$ & $700(200-1,540)$ & $900(300-1,900)$ & $970(200-1,570)$ & 0.009 \\
\hline $\begin{array}{l}\text { Total blood loss to drains (mL) } \\
\text { and hidden blood loss }\end{array}$ & $510(0-1,839)$ & $523(114-1,507)$ & $859(120-2,024)$ & $995(84-1,666)$ & 0.003 \\
\hline $\begin{array}{l}\text { Total blood loss }(\mathrm{mL}) \text {, (peroper. } \\
+ \text { drainage) and hidden blood loss }\end{array}$ & $810(300-2,339)$ & $752(326-2,294)$ & $1,159(420-2,424)$ & $1,290(284-2,166)$ & 0.005 \\
\hline
\end{tabular}

$P$ - significance value, median (min-max); IV1 - intravenous administration - single dose; IV2 - intravenous administration - two doses; COMB - combination of intravenous and topical administration; TOP - only topical administration; TKA - total knee arthroplasty
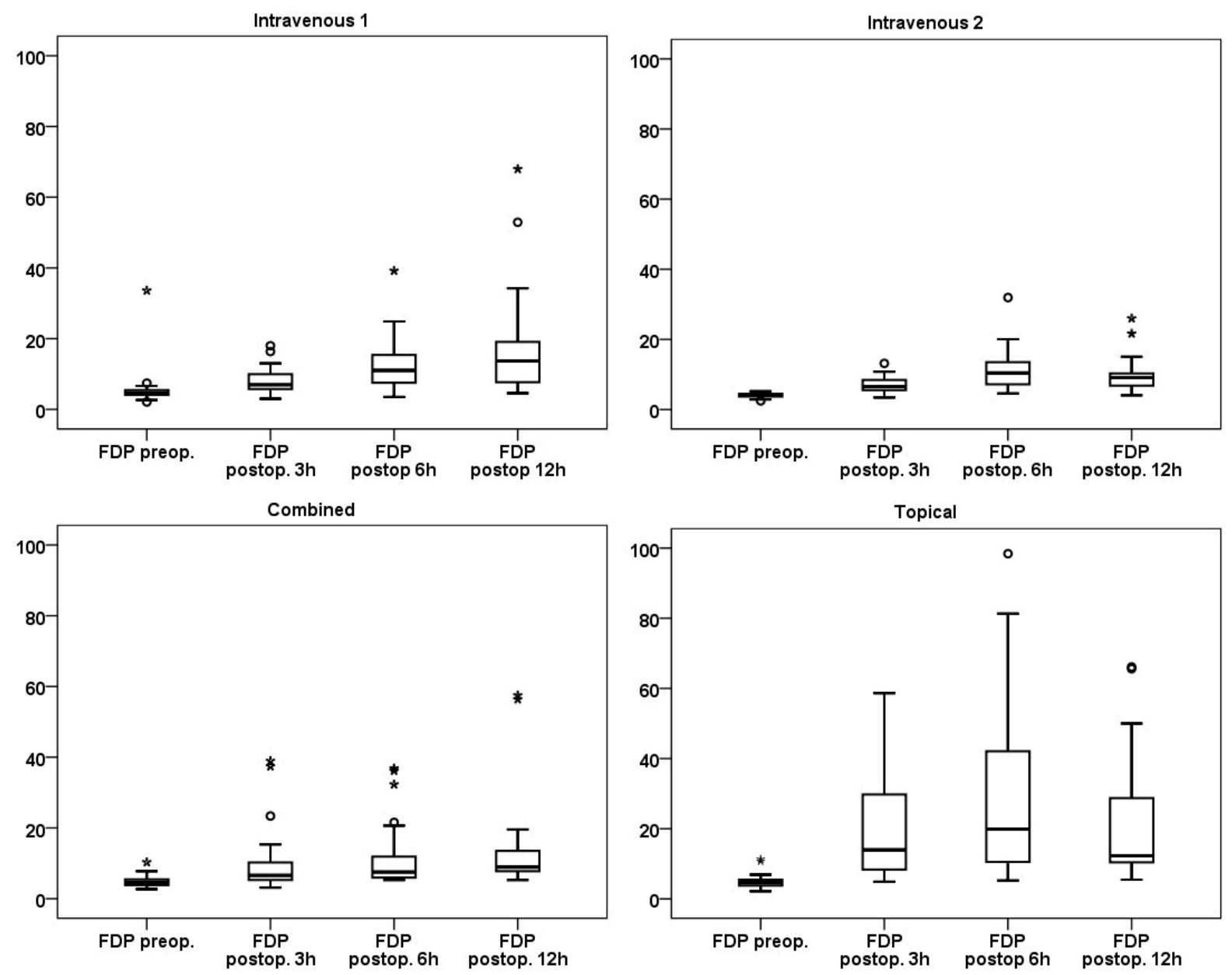

Fig. 2. Comparison of FDP for each group after 3, 6 and $12 \mathrm{~h}$ after the start of the surgery. FDP - fibrin degradation products

\section{RESULTS}

Primary study objective - evaluation of the antifibrinolytic effect of TXA

The pre-operative values of plasminogen, D-dimers and FDP corresponded to variability within the physiological standard (although in one patient we captured HF pre-operatively).

\section{Postoperative level of FDP and D-dimers}

In the course of surgery, the level of fibrinolysis fission products is increased due to tissue damage during surgery. In the first 6 hours after the start of surgery, we observed significantly lower levels of fibrin cleavage products (D-dimers, FDP) in protocols where TXA was administered intravenously or in combination with topical administration (IV1, IV2, COMB) versus topical TXA, which had the least effect on systemic inhibition of fibri- 

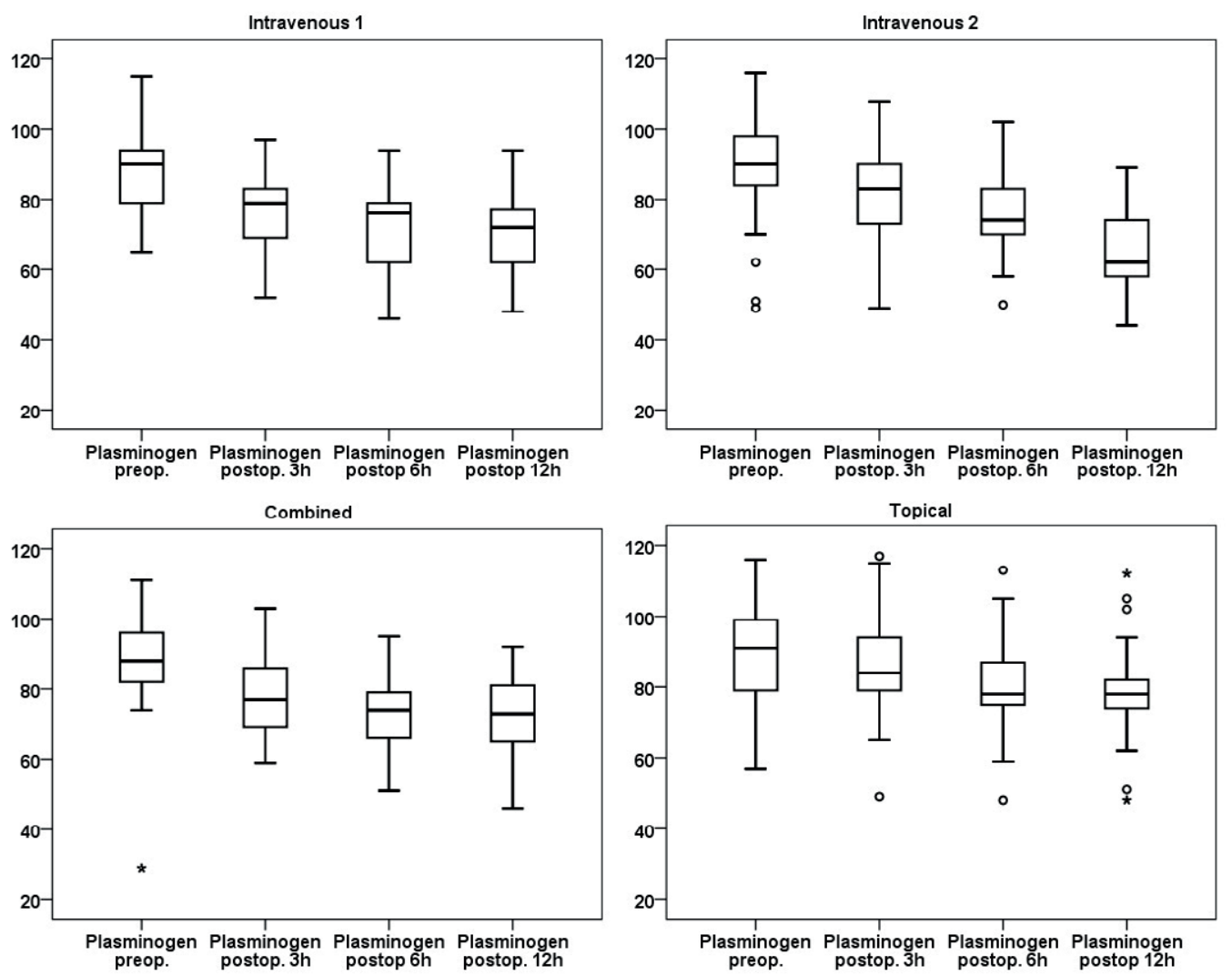

Fig. 3. Comparison of postoperative level of plasminogen at 3,6, and $12 \mathrm{~h}$ after the start of the surgery.
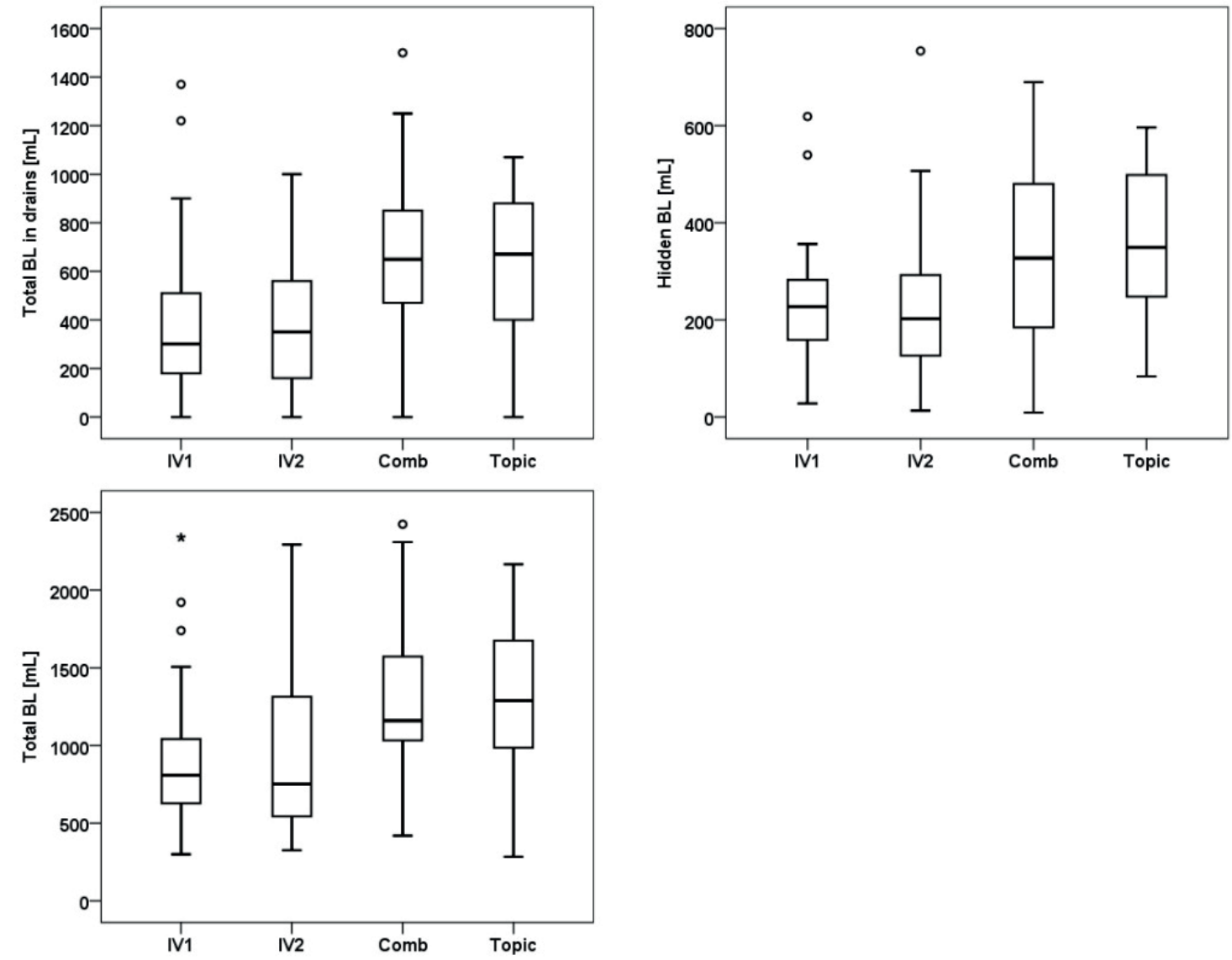

Fig. 4. Range of blood loss for each TXA group.

BL - blood loss; TXA - tranexamic acid; IV1 - intravenous administration - single dose; IV2 - intravenous administration - two doses; Comb - combination of intravenous and topical administration; Topic - only topical administration 
nolysis in all monitored parameters. Table 3 and Fig. 1 and 2 show the basic characteristics and comparison of the individual parameters at each recorded perioperative time.

\section{Plasminogen}

A postoperative plasminogen decrease after intravenous administration of TXA (including combined) occurred over time at each measurement, whereas in topical administration the decrease was only 6 and 12 hours after the start of surgery (Fig. 3). Nevertheless, the fibrinolytic system was never depleted.

Pre-operative plasminogen, respectively 3, 6 and 12 hours after the start of surgery, weakly correlated with age ( $r=-0.201$ to -0.275$)$, while pre-operative plasminogen weakly correlated with BMI ( $\mathrm{r}=0.258)$. The level of preoperative D-dimers weakly correlated with age $(r=0.225)$. Conversely, we did not prove the effect of chosen VTE prevention or smoking at the time of surgical intervention.

\section{Secondary objectives of the study \\ Blood loss including hidden blood loss}

From the point of view of the amount of postoperative blood loss, a significant difference was confirmed between the observed patient groups $(P=0.002)$. Patients with intravenous TXA (IV2, IV1) had the lowest total drainage blood loss compared to patients with TXA topical administration $(P=0.024$ or $P=0.026)$. Intravenous administration (IV1, IV2) also significantly reduced the amount of hidden blood loss compared to topical administration alone. A significant difference in the amount of blood loss was observed 6 hours after the start of surgery $(P=0.001)$ at the earliest. After $12 \mathrm{~h}$, the difference in blood loss was even more pronounced $(P<0.0001)$. When compared to the individual groups in the study, the lowest blood loss was achieved with IV1, IV2 < COMB < TOP regimens. Blood loss during TKA did not differ among the TXA regimens $(P=0.959)$. A summary of the determined results is given in Table 4 and Fig. 4.

\section{Consumption of blood transfusions}

Although the differences in blood loss between the tested TXA regimens were significant, we did not find any differences in blood transfusion consumption $(P=0.410)$.

\section{Decrease in haemoglobin levels}

The lowest decrease in post-operative haemoglobin levels was for combined and topical administration of TXA. Conversely, according to the value of haematocrit or platelets, the preferred regimen of TXA administration could not be identified as most beneficial.

\section{Complications}

There were no differences in the parameters of haematoma, wound secretion, limb swelling, early postoperative revision, or length of hospitalisation among the monitored groups.

\section{DISCUSSION}

Our study first describes the effect of different administration of TXA on the plasma levels of plasminogen, D-dimers and FDP in patients undergoing TKA surgery. From the patient's point of view, it is most important that the treatment or prevention strategies work well, while also being safe at the same time. The first part of the claim is well documented when using TXA in the primary TKA. However, some concerns remain about the unselected systemic use of antifibrinolytic agents. In our study, we found that no intervention significantly interfered with processes associated with systemic fibrinolysis, although D-dimers and FDPs increased over the first 12 hours after the start of surgery and culminated around 6 hours after the start of surgery. The lowest blood loss was found in intravenous TXA regimens, which also had lower drainage. On the contrary, we did not notice differences in blood transfusion consumption.

The effect of TXA varies with its route of administration. The advantage of intravenous TXA administration is its distribution to the extracellular and intracellular space $^{13}$. However, this is at the cost of a higher systemic dose that might at least potentially induce VTE in some patients. A recent meta-analysis, which was aimed, among other things, at determining the safety profile of systemic and topical administration of TXA, did not reveal a higher risk of VTE in either of the analysed modes of administration $^{24}$. No available evaluated study showed a higher incidence of VTE compared to placebo and routine surgical haemostasis. Intravenous administration of TXA did not increase the risk of VTE, neither in patients who had already experienced this complication ${ }^{25}$. Similarly, administration of TXA does not increase 30-day mortality ${ }^{26}$. The explanation will probably be comprehensive. Apparently, in this case, there is a strong "balancing" effect on the area-wide prevention of VTE, which is now recommended after implantation of TKA. However, it is also important that we did not detect significant interference with the fibrinolytic system from the fibrinolysis markers point of view.

The TXA effect should depend on the dose and duration of TXA administration. Therefore, with the intravenous administration of two doses of TXA, we should expect a deeper antifibrinolytic effect with a clear impact on the overall size of blood loss, the size of hidden blood loss, or the decrease in haemoglobin levels. Although a recent meta-analysis does not report a profound effect of multiple TXA administration ${ }^{11}$, randomised clinical trials clearly demonstrated the clinical utility of multiple intravenous TXA administration ${ }^{27,28}$. As of the date of writing, only a few pharmacokinetic studies have been undertaken to assess the impact of TXA on the antifibrinolytic system in detail. In one recent study with oral TXA (ref. $\left.{ }^{3}\right)$, the plasma levels of TXA after oral administration achieved the threshold for the haemostatic effect $(10 \mathrm{mg} / \mathrm{L})$ at 10 , 14,18 and $22 \mathrm{~h}$ after surgery, and for all of the examined modes (i.e., 1, 2, 3 or 4 doses). A similar arrangement as in our study was also used by Zhang et al., who observed 
D-dimer and FDP pre-operatively, respectively on the $1^{\text {st }}$ and $3^{\text {rd }}$ post-operative day after intravenous, combined and topical administration of TXA. They found that the greatest decrease in D-dimers and FDP occurred with combined administration compared to intravenous and topical $^{29}$. We monitored a substantially shorter postoperative period and detected the lowest D-dimer and FDP increase in intravenous component regimens, while topical application of TXA had the lowest systemic antifibrinolytic effect (i.e., the level of D-dimer and FDP were highest). Similar dynamics were presented by other authors who postoperatively captured a slightly larger decrease in D-dimers in TXA patients than in the non-TXA group ${ }^{30}$. Pong et al. observed the development of D-dimers and fibrinogen levels in spinal operations with and without TXA application. In TXA-treated patients, the post-operative D-dimer level was approximately three-fold lower than those performed without TXA (ref. ${ }^{31}$ ).

Fibrinolysis is a physiological and highly regulated response of the organism to the formation of fibrin deposits occurring during vascular wall injury ${ }^{32}$. The principle of fibrinolysis is the cleavage of the fibrin network, which is the basic building block of the blood clot, by the action of the enzyme plasmin. Plasmin works as a serine protease cleaving high molecular weight fibrin polymers to fragments of varying sizes. Fibrinolysis products, either DDIM (specific fibrin fibre cleavage product) or FDP (non-specific fibrinogen and fibrin fibre cleavage products) provide us with information about the residual activity of plasmin when inhibiting with TXA. These markers may be affected by a number of other factors, ranging from basal fibrinogen levels, but also by the extent and duration of surgery. However, their change over time is already significant for the HF course in the postoperative period.

Plasminogen level monitoring is an important parameter of fibrinolysis activity blocked by TXA administration. In case of insufficient inhibition of fibrinolysis, plasma plasminogen levels decrease as a result of its conversion to plasmin, which is undesirable. Small changes in plasma levels indicate, among others, the safe effect of TXA administration. Godier et al. evaluated the in vitro plasminogen activator (t-PA) and TXA effect on blood coagulation and fibrinolysis. They state that TXA in the cascade does not affect plasmin activation, but rather inhibits fibrinolysis by protecting fibrinogen from fibrinogenolysis ${ }^{33}$. Tests such as the determination of plasmin-antiplasmin complexes or alpha 2-antiplasmin assays are time-consuming and difficult to perform in everyday clinical work. The same applies to the euglobulin lysis period, long regarded as a gold standard, but also time-consuming and prone to error ${ }^{1}$. In viscoelastic tests such as thromboelastometry or thrombelastography (Rote TEG), on the other hand, the HF can be detected, but with a significantly lower sensitivity compared with DDIM and FDP, and only when the level of plasmin-antiplasmin complex is very high or the alpha 2-antiplasmin level is very low ${ }^{34}$.

The main effect of TXA administration is a reduction in blood loss, which should automatically lead to a less frequent indication of blood transfusions ${ }^{24}$. Regarding the primary effect of TXA intervention, the recent metaanalysis shows the lowest risk of blood transfusion in combined TXA administration compared to intravenous, IV and purely local p.o. ${ }^{24}$. We failed to prove a significant reduction in the consumption of blood trasnfusions when comparing the study groups. In spite of all standardisation attempts ${ }^{35}$, the indication of blood transfusion is still subject to some variability in physician decision making, and it is therefore not easy to link it with preventive strategies. Although our study was not primarily designed to demonstrate the main effect of TXA administration, we were able to detect a reduction in blood loss, which only illustrates the strength of the intervention being evaluated. Similarly, our study was not primarily focused on finding complications potentially related to TXA administration. Here we found the smallest incidence of complications with intravenous TXA regimens and a combined mode. In this case, the results correspond to large studies, respectively meta-analyses ${ }^{10,11,13}$.

\section{Study limitations}

The present study has several limitations. In the first place, low numbers of patients may be criticised in each arm of the study. However, the primary objective was to assess the effect of TXA administration on hyperfibrinolysis. Another complaint may be directed to a short postoperative follow-up of fibrinolysis (only $12 \mathrm{~h}$ ). We believe that this time is adequate to identify trends in the development of hyperfibrinolysis after TXA administratered during surgery or 6 hours after. We are also aware of the fact that, in particular, when calculating peroperative blood losses, it can only be a gross estimate. There is no validated protocol to monitor and accurately measure the volume of blood in masks, covers, waste, etc. Another limitation of our study is slight ambiguity in the physicians' approach to post-operative blood loss. Some indicate the administration of allogeneic blood at haemoglobin of 95-99 g/L, while others with a blood supply under certain circumstances (younger patients without signs of anaemic syndrome, etc.) wait for a level of $90 \mathrm{~g} / \mathrm{L}$ or less. So theoretically, saving blood transfusions could be even higher.

\section{CONCLUSION}

Our study demonstrated a relatively low influence of all the TXA regimens on systemic markers of fibrinolysis in patients undergoing TKA. From this point of view, TXA administration in TKA can be considered safe as they should not induce undesirable consequences associated with fibrinolysis disruption. While on the other hand the topical administration of TXA was least effective, especially in terms of reduced blood loss (this assertion should be taken at the level of the secondary outcome). None of the tested regimens was associated with a higher incidence of local complications. 
Acknowledgement: The study was supported by Ministry of Health, Czech Republic - conceptual development of research organization (FNOL, 00098892).

Author contributions: JL, JG: conception and study design; JL, LS, LB: collection of samples, clinical data and interpretation; JZ: statistical analysis; JG, JL, LS: manuscript preparation; JG: critical revision.

Conflict of interest: The authors state that there are no conflicts of interest regarding the publication of this article.

\section{REFERENCES}

1. Lee SY, Chong S, Balasubramanian D, Na YG, Kim TK. What is the Idea Route of Administration of Tranexamic Acid in TKA? A Randomized Controlled Trial. Clin Orthop Relat Res 2017;475(8):1987-96.

2. Pabinger I, Fries D, Schochl H, Streif W, Toller W. Tranexamic acid for treatment and prophylaxis of bleeding and hyperfibrinolysis. Wien Klin Wochenschr 2017;129(9-10):303-16.

3. Wang D, Luo ZY, Yu ZP, Liu LX, Chen C, Meng WK, Yu QP, Pei FX, Zhou ZK, Zeng WN. The antifibrinolytic and anti-inflammatory ef fects of multiple doses of oral tranexamic acid in total knee arthroplasty patients: a randomized controlled trial. J Thromb Haemost 2018;16(12):2442-53.

4. Churchill JL, Puca KE, Meyer E, Carleton M, Anderson MJ. Comparing epsilon-Aminocaproic Acid and Tranexamic Acid in Reducing Postoperative Transfusions in Total Knee Arthroplasty. J Knee Surg 2017;30(5):460-6.

5. Fillingham $Y A$, Ramkumar DB, Jevsevar DS, Yates AJ, Shores $P$, Mullen K, Bini SA, Clarke HD, Schemitsch E, Johnson RL, Memtsoudis SG, Sayeed SA, Sah AP, Della Valle CJ. The Efficacy of Tranexamic Acid in Total Knee Arthroplasty: A Network Meta-Analysis. J Arthroplasty 2018;33(10):3090-8.e1.

6. Kopanidis P, Hardidge A, McNicol L, Tay S, McCall P, Weinberg L. Perioperative blood management programme reduces the use of allogenic blood transfusion in patients undergoing total hip and knee arthroplasty. J Orthop Surg Res 2016;11:28.

7. Mi B, Liu G, Zhou W, Lv H, Liu Y, Zha K, Wu Q, Liu J. Intra-articular versus intravenous tranexamic acid application in total knee arthroplasty: a meta-analysis of randomized controlled trials. Arch Orthop Trauma Surg 2017;137(7):997-1009.

8. Chen TP, Chen YM, Jiao JB, Wang YF, Qian LG, Guo Z, Ma Z, Han CY, Shi TH. Comparison of the effectiveness and safety of topical versus intravenous tranexamic acid in primary total knee arthroplasty: a meta-analysis of randomized controlled trials. J Orthop Surg Res 2017;12(1):11.

9. Alshryda S, Sukeik M, Sarda P, Blenkinsopp J, Haddad FS, Mason JM. A systematic review and meta-analysis of the topical administration of tranexamic acid in total hip and knee replacement. Bone Joint J 2014;96-b(8):1005-15.

10. Xiong $\mathrm{H}$, Liu $\mathrm{Y}$, Zeng $\mathrm{Y}, \mathrm{Wu} \mathrm{Y}$, Shen B. The efficacy and safety of combined administration of intravenous and topical tranexamic acid in primary total knee arthroplasty: a meta-analysis of randomized controlled trials. BMC Musculoskelet Disord 2018;19(1):321.

11. Sun Q, Li J, Chen J, Zheng C, Liu C, Jia Y. Comparison of intravenous, topical or combined routes of tranexamic acid administration in patients undergoing total knee and hip arthroplasty: a meta-analysis of randomised controlled trials. BMJ Open 2019;9(1):e024350.

12. Han YH, Huang HT, Pan JK, Zeng LF, Liang GH, Liang HD, Yang WY, Guo D, Liu J. Is the combined application of both drain-clamping and tranexamic acid superior to the single use of either application in patients with total-knee arthroplasty?: A meta-analysis of randomized controlled trials. Medicine 2018;97(36):e11573.

13. Xie J, Hu Q, Huang Z, Zhou Z, Pei F. Comparison of three routes of administration of tranexamic acid in primary unilateral total knee arthroplasty: Analysis of a national database. Thromb Res 2019;173:96101.

14. Kim YT, Kang MW, Lee JK, Lee YM, Kim Jl. Combined use of topical intraarticular tranexamic acid and rivaroxaban in total knee ar- throplasty safely reduces blood loss, transfusion rates, and wound complications without increasing the risk of thrombosis. BMC Musculoskelet Disord 2018;19(1):227.

15. Good L, Peterson E, Lisander B. Tranexamic acid decreases externa blood loss but not hidden blood loss in total knee replacement. $\mathrm{Br}$ J Anaesth 2003;90(5):596-9.

16. Edy J, De Cock F, Collen D. Inhibition of plasmin by normal and antiplasmin-depleted human plasma. Thromb Res 1976;8(4):513-8.

17. Teger-Nilsson AC, Friberger $P$, Gyzander E. Determination of a new rapid plasmin inhibitor in human blood by means of a plasmin specific tripeptide substrate. Scand J Clin Lab Invest 1977;37(5):403-9.

18. Frieberger P. Methods for the determination of plasmin, antiplasmin and plasminogen by means of the substrate. Haemostasis 1975;7:138-45.

19. Gaffney PJ. The occurrence and clinical relevance of fibrin fragments in blood. Ann NY Acad Sci 1983;408:407-23.

20. Olson JD. D-dimer: An Overview of Hemostasis and Fibrinolysis, Assays, and Clinical Applications. Adv Clin Chem 2015;69:1-46.

21. Levi M. Diagnosis and treatment of disseminated intravascular coagulation. Int J Lab Hematol 2014;36(3):228-36.

22. Newman DJ, Henneberry H, Price CP. Particle enhanced light scattering immunoassay. Ann Clin Biochem 1992;29 ( Pt 1):22-42.

23. Palareti G. Fibrinogen/fibrin degradation products: Pathophysiology and clinical application. Fibrinolysis 1993;7:60-1.

24. Xu S, Chen JY, Zheng Q, Lo NN, Chia SL, Tay KJD, Pang HN, Shi L, Chan ESY, Yeo SJ. The safest and most efficacious route of tranexamic acid administration in total joint arthroplasty: A systemic review and network meta-analysis. Thromb Res 2019;176:61-6.

25. Sabbag OD, Abdel MP, Amundson AW, Larson DR, Pagnano MW. Tranexamic Acid Was Safe in Arthroplasty Patients With a History of Venous Thromboembolism: A Matched Outcome Study. J Arthroplasty 2017;32(9S):S246-S50.

26. Duncan CM, Gillette BP, Jacob AK, Sierra RJ, Sanchez-Sotelo J, Smith HM. Venous thromboembolism and mortality associated with tranexamic acid use during total hip and knee arthroplasty. J Arthroplasty 2015;30(2):272-6.

27. Xie J, Ma J, Yao H, Yue C, Pei F. Multiple Boluses of Intravenous Tranexamic Acid to Reduce Hidden Blood Loss After Primary Total Knee Arthroplasty Without Tourniquet: A Randomized Clinical Trial. J Arthroplasty 2016;31(11):2458-64.

28. Lei Y, Xie J, Xu B, Xie X, Huang Q, Pei F. The efficacy and safety of multiple-dose intravenous tranexamic acid on blood loss following total knee arthroplasty: a randomized controlled trial. Int Orthop 2017;41(10):2053-9.

29. Zhang YM, Yang B, Sun XD, Zhang Z. Combined intravenous and intra-articular tranexamic acid administration in total knee arthroplasty for preventing blood loss and hyperfibrinolysis: A randomized controlled trial. Medicine (Baltimore) 2019;98(7):e14458.

30. Burleson A, Guler N, Banos A, Syed D, Wanderling C, Hoppensteadt D, Rees H, Fareed J, Hopkinson W. Perioperative Factors and Their Effect on the Fibrinolytic System in Arthroplasty Patients. Clin Appl Thromb Hemost 2016;22(3):274-9.

31. Pong RP, Leveque JA, Edwards A, Yanamadala V, Wright AK, Herodes M, Sethi RK. Effect of Tranexamic Acid on Blood Loss, D-Dimer, and Fibrinogen Kinetics in Adult Spinal Deformity Surgery. J Bone Joint Surg Am 2018;100(9):758-64.

32. Picetti R, Shakur-Still H, Medcalf RL, Standing JF, Roberts I. What concentration of tranexamic acid is needed to inhibit fibrinolysis? A systematic review of pharmacodynamics studies. Blood Coagul Fibrinolysis 2019;30(1):1-10.

33. Godier A, Parmar K, Manandhar K, Hunt BJ. An in vitro study of the effects of t-PA and tranexamic acid on whole blood coagulation and fibrinolysis. J Clin Pathol 2017;70(2):154-61.

34. Raza I, Davenport R, Rourke C, Platton S, Manson J, Spoors C Khan S, De'Ath HD, Allard S, Hart DP, Pasi KJ, Hunt BJ, Stanworth S, MacCallum PK, Brohi K. The incidence and magnitude of fibrinolytic activation in trauma patients. J Thromb Haemost 2013;11(2):307-14.

35. Carson JL, Stanworth SJ, Alexander JH, Roubinian N, Fergusson DA, Triulzi DJ, Goodman SG, Rao SV, Doree C, Hebert PC. Clinical trials evaluating red blood cell transfusion thresholds: An updated systematic review and with additional focus on patients with cardiovascular disease. Am Heart J 2018;200:96-101. 